\title{
Transport coefficients for dense hard-disk systems
}

\author{
Ramón García-Rojo \\ Institute for Computational Physics, University of Stuttgart, Pfaffenwaldring 27, 70569 Stuttgart, Germany
}

Stefan Luding

Technische Universiteit Delft (TUD), DelftChemTech, Particle Technology, Nanostructured Materials, Julianalaan 136, 2628 BL Delft, The Netherlands

J. Javier Brey

Física Teórica, Universidad de Sevilla, Apartado de Correos 1065, E-41080 Sevilla, Spain

(Received 28 November 2005; revised manuscript received 8 November 2006; published 13 December 2006)

\begin{abstract}
A study of the transport coefficients of a system of elastic hard disks based on the use of Helfand-Einstein expressions is reported. The self-diffusion, the viscosity, and the heat conductivity are examined with averaging techniques especially appropriate for event-driven molecular dynamics algorithms with periodic boundary conditions. The density and size dependence of the results are analyzed, and comparison with the predictions from Enskog's theory is carried out. In particular, the behavior of the transport coefficients in the vicinity of the fluid-solid transition is investigated and a striking power law divergence of the viscosity with density is obtained in this region, while all other examined transport coefficients show a drop in that density range in relation to the Enskog's prediction. Finally, the deviations are related to shear band instabilities and the concept of dilatancy.
\end{abstract}

DOI: 10.1103/PhysRevE.74.061305

PACS number(s): 05.60.-k, 02.70.Ns, 05.20.Dd

\section{INTRODUCTION}

Transport coefficients characterize the different dissipation mechanisms in nonequilibrium states. At the macroscopic level, they are introduced by phenomenological equations, like the Navier-Stokes equations for a simple fluid, which predict the time evolution of mass, momentum, and energy [1]. Each transport coefficient is related to the propagation of one (or more) of these microscopic quantities, bridging therefore the hydrodynamic and the microscopic scale. In the case of low density gases, the macroscopic equations have been justified, their range of validity has been determined, and explicit expressions for the transport coefficients have been obtained using the Boltzmann kinetic equation as the starting point [2,3]. At higher but moderate densities, the Enskog equation has also proved to give a quite accurate description of a gas of hard spheres or disks.

A remarkable and fundamental development in the statistical mechanics theory of transport processes was the derivation of expressions for the transport coefficients based on equilibrium time-correlation functions. These are the socalled Green-Kubo formulas, and they involve different microscopic fluxes [4]. These expressions, although formal, are of general validity and have been extensively used for the analysis and modeling of transport in dense systems. In particular, they have been applied to the computation of transport coefficients by means of molecular dynamics simulations.

Alternative formal expressions for the transport coefficients are provided by the Einstein-Helfand formulas $[5,6]$, the simplest of which being Einstein's formula for the selfdiffusion coefficient in terms of the second moment of the displacements. The Einstein-Helfand expressions for the other transport coefficients involve moments of corresponding dynamical variables, which are the time integrals of the microscopic fluxes appearing in the Green-Kubo relations.

In the last years, there has been a revived interest in transport processes in systems composed by hard particles motivated by the study of granular media in general, and granular gases as a special case $[7-9,19,50]$. The simplest valid model of them is an assembly of inelastic hard spheres or disks, in which the inelasticity is accounted for only through one constant parameter, the coefficient of normal restitution. In the low density limit, hydrodynamic Navier-Stokes like equations, with explicit expressions for the transport coefficients, have been obtained for this model, by starting from the inelastic extension of the Boltzmann equation [10]. Moreover, it has been shown that the transport coefficients for a dilute granular gas can be expressed in the form of generalized Green-Kubo relations $[11,12]$.

The Enskog equation has also been extended to inelastic particles $[13,14]$, but its density and inelasticity range of validity is still not clear. On the other hand, formally exact relations between transport coefficients and appropriate correlation functions, similar to the Green-Kubo and EinsteinHelfand formulas, appear up to now limited to the low density limit mentioned above and to the simplest cases of tagged particle motion $[15,16]$, although there have been some more general proposals $[17,18]$. Therefore, for high densities, the only available hydrodynamic theory for granular systems is restricted to the so-called quasielastic limit.

The first calculations of transport coefficients for hardsphere systems by means of equilibrium molecular dynamics simulations go back to the pioneering works by Alder and co-workers [20,21]. The dependence of the values of the transport coefficients on the density and also on the number of particles used in the simulations have been analyzed. At high densities, significant deviations from the Enskog theory 
predictions are observed, especially for the self-diffusion and shear viscosity coefficients [22-25].

In spite of all the work done for three dimensional systems, it is hard to find results for two dimensions, i.e., for a fluid of hard disks. A notable exception is Ref. [26], where the viscosity of a system of hard disks is measured by using an Einstein-Helfand expression. It could be argued that this is due to the presence of long time tails in the equilibrium correlation functions appearing in the Green-Kubo expressions of the transport coefficients [27,28,51], but it must be noticed that they do not invalidate by themselves the possibility of a hydrodynamic description. Mode coupling theory and, more conclusively, simulation results obtained in latticegas cellular automata have shown that the asymptotic logarithmic tail is expected to dominate only on time scales unphysically large, and therefore irrelevant from the practical point of view. In this study the logarithmic correction will be consequently ignored, and effective values of the transport coefficients will be calculated and compared with Enskog predictions. A more detailed discussion of this point will be given in later sections of the paper.

In this paper, the transport coefficients of a system of hard disks will be measured by means of Einstein-Helfand expressions that are appropriate for molecular dynamics simulations with periodic boundary conditions, using the minimum image convention [6]. For continuous interaction potentials, this method is strictly equivalent to the Green-Kubo method and has the advantages of directly showing the positivity of the transport coefficients and of being based on a straightforward, numerically robust accumulation [26]. In the case of hard particles, there is a fundamental reason to use methods based on Helfand-Einstein expressions for the transport coefficients. The Green-Kubo relations, except in the case of self-diffusion, involve forces between the particles, which are ill-defined for hard spheres or disks and there is no trivial way to extend them to hard-particle fluids. In fact, a recent careful analysis of the dynamics of a system of hard spheres has shown that the correct Green-Kubo expressions for this system have a new singular contribution due to instantaneous collisions, as well as the usual time integral of the flux correlation functions [30]. The singular part vanishes in the low density limit, but gives a relevant contribution at high densities. On the other hand, the Einstein-Helfand formulas do not involve the forces, and have the same form for both continuous (soft) and rigid (hard-sphere) potentials. It must be stressed at this point that it is an Einstein-Helfand method that was already numerically implemented by Alder et al. in their study of the transport coefficients in hard-sphere fluids $[20,21]$.

The most interesting finding of the present study is that the shear viscosity shows a divergence at the crystallization density (in a nonsheared system), while the heat conductivity is correlated to the isotropic pressure. Thus, while pressure and heat conductivity show a small drop at crystallization (due to the better ordering of the particles), selfdiffusion vanishes close to that density, and shear viscosity diverges. This divergence is well below the excluded volume caused divergence of pressure and heat conductivity at the maximum density possible in hard disk systems.

The outline of the paper is as follows. In the Sec. II, the Einstein-Helfand expressions for the transport coefficients are revised, and written in a way that is appropriate for hard sphere molecular dynamics simulations with periodic boundary conditions. The special case of self-diffusion, in which the actual trajectories of the particles along different cells must be followed, is first discussed. For all the other transport coefficients studied here, it is shown that a decomposition of the contributions to the transport coefficients into a kinetic and a collisional part, allows the use of the minimum image convention. Moreover, the decomposition will turn out to be especially useful for event driven simulation algorithms. In Sec. III, the method is applied to a system of hard disks for calculating the self-diffusion, shear viscosity, and heat conductivity coefficients over the whole density range. The results are compared with the theoretical predictions from Enskog's theory. Particular emphasis is put on the behavior of the transport coefficients in the fluid-solid transition region and on characterizing the divergence of the shear viscosity. The paper finishes with the discussion of the main results in Sec. IV.

\section{EINSTEIN-HELFAND EXPRESSIONS FOR THE TRANSPORT COEFFICIENTS}

\section{A. The self-diffusion coefficient}

Self-diffusion is the macroscopic transport phenomenon describing the motion of tagged particles in a fluid at equilibrium, in the limiting case that their concentration is very low, while at the same time they are mechanically identical to the fluid particles. The macroscopic number density $n(\boldsymbol{r}, t)$ and the flux $\boldsymbol{j}(\boldsymbol{r}, t)$ of tagged particles satisfy the continuity equation

$$
\partial_{t} n(\boldsymbol{r}, t)+\boldsymbol{\nabla} \cdot \boldsymbol{j}(\boldsymbol{r}, t)=0 .
$$

The corresponding constitutive relation closing the above equation is provided by Fick's law

$$
\boldsymbol{j}(\boldsymbol{r}, t)=-D \nabla n(\boldsymbol{r}, t),
$$

which defines the self-diffusion coefficient $D$. An expression for this transport coefficient in terms of the second moment of the displacements is given by the well-known Einstein formula $[2,3]$

$$
D=\frac{1}{2 d} \lim _{t \rightarrow \infty} \frac{d}{d t}\left\langle|\boldsymbol{r}(t)-\boldsymbol{r}(0)|^{2}\right\rangle,
$$

where $\boldsymbol{r}(t)$ is the position of an arbitrary tagged particle at time $t, d$ is the dimensionality of the system, and the angular brackets denote an average over the ensemble describing the equilibrium of the system.

To actually compute the above equation in our numerical algorithm, two different averages have been carried out. First, an average over the $N$ particles in the system is taken, and then a second average over a number $\mathcal{N}$ of initial configurations (trajectories). Assuming ergodicity of the system, different trajectories can be generated from the same simulation run by considering different initial times $t_{k}$. Therefore, the full average is 


$$
\left\langle|\boldsymbol{r}(t)-\boldsymbol{r}(0)|^{2}\right\rangle=\frac{1}{N \mathcal{N}} \sum_{k=1}^{\mathcal{N}} \sum_{i=1}^{N}\left|\boldsymbol{r}_{i}\left(t+t_{k}\right)-\boldsymbol{r}_{i}\left(t_{k}\right)\right|^{2} .
$$

This double averaging is possible because the dynamical variable involved in Eq. (3) is a mono-particle property in the present case, and all the particles are equivalent.

When using periodic boundary conditions for evaluating Eq. (4), as in the simulations to be reported here, it is crucial to take into account that particles on a given trajectory, have to be followed as they cross the border of the periodic system. The positions in Eq. (4), as written down here, use the actual positions, but the real relative displacements should be used instead. If periodic images of the center cell were not used, the displacements would not be obtained correctly. In this sense, the practical implementation of the algorithm for this transport coefficient differs from those used for the other transport coefficients to be discussed in the following.

The simulation results for $D$ to be reported later on, will be scaled with the value obtained from the Enskog equation in the first Sonine approximation for $d=2$ that is [31]:

$$
D_{E}=\frac{1}{2 n \sigma g_{2}(\sigma)}\left(\frac{k_{B} T}{\pi m}\right)^{1 / 2} .
$$

Here $T$ is the temperature, $k_{B}$ the Boltzmann constant, $\sigma$ the diameter of the disks, $m$ their mass, and $g_{2}(\sigma)$ the value of the equilibrium pair correlation function at contact, which is a function of the density $n$. An estimate for this quantity is provided by Henderson's expression [32]:

$$
g_{2}(\sigma)=\frac{1-\frac{7 \nu}{16}}{(1-\nu)^{2}},
$$

with $\nu=n \pi \sigma^{2} / 4$ being the solid fraction. The approximation in (6) is valid for densities well below the crystallization solid fraction $\nu_{c} \approx 0.7$.

\section{B. Shear viscosity}

The coefficients of shear viscosity $\eta$ and bulk viscosity $\zeta$ are defined through the macroscopic expression for the pressure tensor $\mathrm{P}$ for a simple fluid [1]

$$
\mathrm{P}(\boldsymbol{r}, t)=p 1-\eta\left[\nabla \boldsymbol{u}+(\boldsymbol{\nabla} \boldsymbol{u})^{+}\right]+\left(\frac{2 \eta}{3}-\zeta\right) 1 \boldsymbol{\nabla} \cdot \boldsymbol{u},
$$

where $p$ is the pressure, $\boldsymbol{u}$ the flow velocity, 1 the unit tensor, and the superscript + means here transposed.

The Einstein-Helfand formulas for $\eta$ and $\zeta$ are analogous to Eq. (3). For the shear viscosity one has $[5,6]$

$$
\eta=\frac{m^{2}}{2 V k_{B} T} \lim _{t \rightarrow \infty} \frac{d}{d t}\left\langle\left|G_{\eta}(t)-G_{\eta}(0)\right|^{2}\right\rangle,
$$

with $V$ the volume of the system, and

$$
G_{\eta}=\sum_{i=1}^{N} \dot{x}_{i} y_{i} .
$$

In this case, only an average over a set of different initial conditions can be taken from the simulations, since the dynamical variable $G_{\eta}$ already involves all the particles in the system. Therefore, the quantity that, in principle, should be obtained from the simulations is

$$
\begin{aligned}
& \left\langle\left|G_{\eta}(t)-G_{\eta}(0)\right|^{2}\right\rangle \\
& \quad=\frac{1}{\mathcal{N}_{k=1}^{N}} \sum^{\mathcal{N}}\left\{\sum_{i=1}^{N}\left[\dot{x}_{i}\left(t+t_{k}\right) y_{i}\left(t_{k}\right)-\dot{x}_{i}\left(t_{k}\right) y_{i}\left(t_{k}\right)\right]\right\}^{2} .
\end{aligned}
$$

Nevertheless, this expression leads to very noisy results and the slope of the best fit line can be only determined with a high uncertainty. Moreover, it presents the same difficulties as the Einstein expression for the self-diffusion coefficient when using periodic boundary conditions, i.e., for the positions $y_{i}$, it requires one to follow the motion of the particles through different unit cells. It is thus convenient to elaborate on Eq. (10) a little more. The idea is measuring the increments of $G_{\eta}$ for physically well defined time intervals, instead of directly determining its actual value at successive times.

Let us consider a time interval $\left[t+t_{k}, t+t_{k}+\Delta t\right]$ in which no collisions occur in the system in a given trajectory $k$. The purely kinetic variation of $G_{\eta}$, due to the displacements only, is

$$
\Delta G_{\eta}^{(K)}\left(t+t_{k}\right)=\sum_{i=1}^{N} \dot{x}_{i}\left(t+t_{k}\right) \dot{y}_{i}\left(t+t_{k}\right) \Delta t .
$$

In addition, there is also a contribution due to the discontinuous change of the velocities in collisions. Consider a collision between particles $i$ and $j$. There is an instantaneous jump in $G_{\eta}$ given by

$$
\Delta G_{\eta}^{(C)}=\dot{x}_{i}^{+} y_{i}+\dot{x}_{j}^{+} y_{j}-\dot{x}_{i}^{-} y_{i}-\dot{x}_{j}^{-} y_{j},
$$

where we have taken into account that the positions do not change during the instantaneous collision and the index + (-) indicates that the velocity is the post-collisional (precollisional) one. Both velocities are related by the collision rule for hard disks

$$
\begin{aligned}
& \dot{\boldsymbol{r}}_{i}^{+}=\dot{\boldsymbol{r}}_{i}^{-}-\dot{\boldsymbol{r}}_{i j} \cdot \hat{\boldsymbol{\sigma}} \hat{\boldsymbol{\sigma}}, \\
& \dot{\boldsymbol{r}}_{j}^{+}=\dot{\boldsymbol{r}}_{j}^{-}+\dot{\boldsymbol{r}}_{i j} \cdot \hat{\boldsymbol{\sigma}} \hat{\boldsymbol{\sigma}},
\end{aligned}
$$

with $\boldsymbol{r}_{i j}=\boldsymbol{r}_{i}-\boldsymbol{r}_{j}$ and $\hat{\boldsymbol{\sigma}}$ being the unit vector joining the centers of disks $i$ and $j$ at contact and pointing from particle $j$ to particle $i$. Using the above rule, Eq. (12) can be rewritten

$$
\Delta G_{\eta}^{(C)}=y_{i j} \delta \dot{x}_{i},
$$

where $y_{i j}=y_{i}-y_{j}$, and $\delta \dot{\boldsymbol{r}}_{i}=-\left(\dot{\boldsymbol{r}}_{i j} \cdot \hat{\boldsymbol{\sigma}}\right) \hat{\boldsymbol{\sigma}}$ is the change of the velocity of particle $i$ in the collision. Since the dynamics of a hard particle system consists of free streaming and instantaneous collisions, Eqs. (11) and (14) fully determine the time evolution of $G_{\eta}$ along a trajectory. Moreover, these equations only involve the velocities and relative positions of the particles. As a consequence, they avoid the difficulties of using other Helfand-Einstein relations with periodic boundary conditions in the simulations, as discussed by Erpenbeck [33], since no contribution leads to the growth in time of the dynamical variable $G_{\eta}$ due to the infinite checkerboard of 
identical systems. This is because contributions from pairs of particles in different unit cells cancel out precisely due to the boundary conditions. An alternative use of a HelfandEinstein relation for computing the shear viscosity with periodic boundary conditions has been discussed in [26].

Equations (11) and (14) are particularly suitable for event driven algorithms as the one employed in the simulations presented in this paper. In these algorithms, the time steps are the intervals between successive instantaneous collisions in the system. At every collision, the kinetic change $\Delta G_{\eta}^{(K)}$ associated with the previous time step is computed as well as the contribution from the collision itself, $\Delta G_{\eta}^{(C)}$. For the latter, only the positions and velocities of the pair of colliding particles must be taken into account, while for the kinetic contribution the motion of all the particles in the system has to be considered.

As for the self-diffusion coefficient, the simulation results for $\eta$ will be reported scaled with the Enskog value in the first Sonine approximation, that for $d=2$ and densities $\nu<\nu_{c}$ is [31]

$$
\eta_{E}=\eta_{0}\left[\frac{1}{g_{2}(\sigma)}+2 \nu+\left(1+\frac{8}{\pi}\right) g_{2}(\sigma) \nu^{2}\right],
$$

where $\eta_{0}$ is the value in the Boltzmann limit

$$
\eta_{0}=\frac{1}{2 \sigma}\left(\frac{m k_{B} T}{\pi}\right)^{1 / 2} \text {. }
$$

\section{Bulk viscosity}

The coefficient of bulk viscosity $\zeta$ was already introduced in Eq. (7). The corresponding Einstein-Helfand expression is $[5,20]$ :

$$
\zeta+\frac{4 \eta}{3}=\frac{m^{2}}{2 V k_{B} T} \lim _{t \rightarrow \infty} \frac{d}{d t}\left\langle\left|G_{\zeta}(t)-G_{\zeta}(0)-\frac{p V t}{m}\right|^{2}\right\rangle,
$$

where

$$
G_{\zeta}=\sum_{i=1}^{N} \dot{x}_{i} x_{i} .
$$

The $p V t$ term in Eq. (17) arises from the fact that the equilibrium average of $G_{\zeta}$ does not vanish, as it is the case for all the other variables $G$ associated to transport coefficients, but it is equal to the external work $p V$, defined by the virial theorem $[5,20]$. Since this mean value is also computed in the simulations and it slightly shifts as the simulations proceed, the results for the right hand side of Eq. (17) are determined much less accurately than for the expressions for the other transport coefficients. Additionally, the subtraction of $\eta$, which itself is determined with some uncertainty, causes further errors in the values estimated for $\zeta$. For these reasons, we have not been able to obtain reliable results for the bulk viscosity in the high density region, and no further consideration will be given to it here. Note however, that it is still possible to split expression (17) in a kinetic and a collisional contribution, like we have done before. For that sake, the microscopic expression of the hydrostatic pressure is used [34]:

$$
p=\frac{m N}{V}\left\langle\dot{x}_{i} \dot{x}_{i}\right\rangle+\frac{m \sigma}{V} \mathcal{F}\left\langle\hat{x}_{i j} \delta \dot{x}_{i}\right\rangle,
$$

where $\hat{x}_{i j}$ is the unitary vector pointing from center of particle $j$ to center of particle $i$, and $\mathcal{F}$ is the collision frequency [2]. In terms of this equation and following the same reasoning as in the previous section, it is straightforward to identify that the kinetic increments on $G_{\zeta}$ in the particular case of the bulk viscosity, are related to deviations of the variable with respect to the mean value. This happens for the kinetic part,

$$
\Delta G_{\zeta}^{(K)}(t+\Delta t)=\sum_{i=1}^{N}\left[\dot{x}_{i}(t) \dot{x}_{i}(t)-\left\langle\dot{x}_{i}(t) \dot{x}_{i}(t)\right\rangle\right] \Delta t
$$

as well as for the collisional part:

$$
\Delta G_{\zeta}^{(C)}(t+\Delta t)=\left[x_{i j} \delta \dot{x}_{i}-\left\langle x_{i j} \delta \dot{x}_{i}\right\rangle\right],
$$

with $\delta \dot{x}_{i}$ being the change of velocity of the particle $i$ in its collision with particle $j$, as already defined above. Note that expressions (20) and (21) are again perfectly compatible with the minimum image convection.

We finally reproduce, for the sake of completeness, the Enskog value of the bulk viscosity for hard disks [31]:

$$
\zeta_{E}=\frac{8 \nu^{2} g_{2}(\sigma)}{\pi \sigma}\left(\frac{m k_{B} T}{\pi}\right)^{1 / 2} .
$$

\section{Thermal conductivity}

The coefficient of thermal conductivity $\lambda$ is defined by the Fourier law for the heat flux $\boldsymbol{q}(\boldsymbol{r}, t)[1]$

$$
\boldsymbol{q}(\boldsymbol{r}, t)=-\lambda \boldsymbol{\nabla} T(\boldsymbol{r}, t) .
$$

Enskog's theory prediction for the heat conductivity is

$$
\lambda_{E}=\lambda_{0}\left[\frac{1}{g_{2}(\sigma)}+3 \nu+\left(\frac{9}{4}+\frac{4}{\pi}\right) \nu^{2} g_{2}(\sigma)\right],
$$

where $\lambda_{0}$ is the Boltzmann value

$$
\lambda_{0}=\frac{2 k_{B}}{\sigma}\left(\frac{k_{B} T}{\pi m}\right)^{1 / 2} .
$$

The Einstein-Helfand expression for this coefficient is [23]

$$
\lambda=\frac{1}{2 V k_{B} T^{2}} \lim _{t \rightarrow \infty} \frac{d}{d t}\left\langle\left|G_{\lambda}(t)-G_{\lambda}(0)\right|^{2}\right\rangle,
$$

with

$$
G_{\lambda}=\sum_{i=1}^{N} x_{i} e_{i}
$$

Here $e_{i}$ is the energy of particle $i$, 


$$
e_{i}=\frac{m \dot{\boldsymbol{r}}_{i}^{2}}{2}
$$

As it was the case with Eq. (10), also Eq. (26) is not well suited for numerical simulations with periodic boundary conditions, since it involves the positions of the particles. Therefore, we are going to transform it in a similar way, i.e., measuring separately the increments of the dynamical variable $G_{\lambda}$ between collisions and the collisional contributions. The variation of $G_{\lambda}$ along a trajectory $k$ in a time interval $\Delta t$ such that no collisions take place is

$$
\Delta G_{\lambda}^{(K)}=\frac{1}{2} \sum_{i=1}^{N}\left[\dot{x}_{i}\left(t+t_{k}\right) e_{i}\left(t+t_{k}\right)-\dot{y}_{i}\left(t+t_{k}\right) e_{i}\left(t+t_{k}\right)\right],
$$

where the isotropy of the equilibrium state has been taken into account. The statistical quality of the simulation results is therefore increased.

In a collision between particles $i$ and $j$, the instantaneous change in $G_{\lambda}$ is

$$
\Delta G_{\lambda}^{(C)}=x_{i} e_{i}^{+}+x_{j} e_{j}^{+}-x_{i} e_{i}^{-}-x_{j} e_{j}^{-}=x_{i j}\left(e_{i}^{+}-e_{i}^{-}\right)=x_{i j} \delta e_{i} .
$$

The second equality follows from the total energy conservation in the collisions here considered. Using again the isotropy of the system, we can write

$$
\Delta G_{\lambda}^{(C)}=\frac{\left(x_{i j}+y_{i j}\right) \delta e_{i}}{2} .
$$

Equations (29) and (31) do not contain absolute positions of the particles, but only their relative positions and their velocities. Therefore, we have obtained again an expression that does not require to follow the track of the particles all along the simulation, namely to use the so-called unfolded particle coordinates. The values of the position and velocities of the particles expressed in folded coordinates can be used, without taking care of the cell crossing.

\section{E. Qualitative discussion}

In this subsection we briefly discuss some issues in a qualitative manner, before we analyze and discuss simulation data in the next section and show quantitative results.

The decomposition of the dynamical variables and the methods explained above in this section, are validated for a wide density range below. The time evolution of the dynamical variables in Eqs. (4), (9), and (27), is linear in the whole range of densities and already from a very small number of Enskog collision times on. The values of the transport coefficients can be therefore easily calculated from the slope of the different curves, as a consequence of Eqs. (3), (8), and (26). Using as an example the mean square displacement (self-diffusion), this is illustrated below, see Fig. 1. Similar results are found for the other transport coefficients (data not shown).

The effect of the time step for the analysis of the numerical simulation will also be discussed below, see Fig. 7.

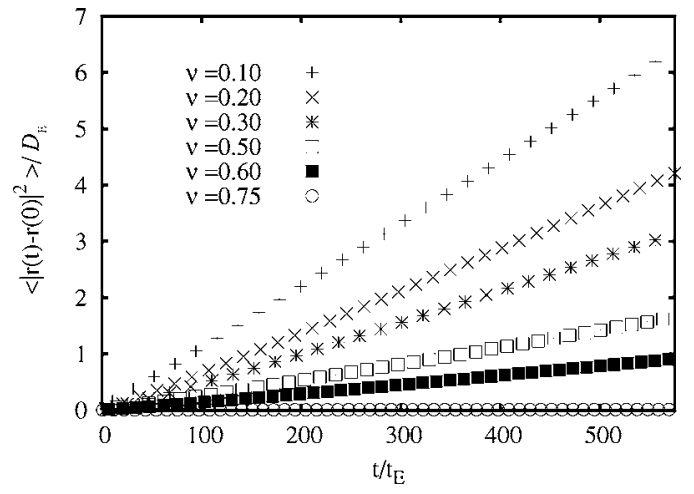

FIG. 1. Time evolution of the mean square displacement (scaled with Enskog prediction for the self-diffusion coefficient $D_{E}$ ) for several densities ranging from the dilute $(\nu=0.1)$ to the dense limit $(\nu=0.75)$. The time is scaled with the Enskog collision time.

Qualitatively speaking, the optimal value for $\Delta t$ should be below the Enskog collisional time, in order to obtain a correct value for the transport coefficients. There should be maximally one collision for any particle within one averaging time step $\Delta t$. On the other hand it is inefficient to make $\Delta t$ too small, since this slows down the simulation scheme.

The relative weight of the collisional and kinetic contributions on the overall value of, e.g., the thermal conductivity transport coefficient, trivially depends on the density of the system. Below, in Fig. 8, it will be shown that the ratio of kinetic to collisional contribution decreases exponentially fast as density increases. The kinetic contribution is a determinant for very dilute systems, but as density increases the collisional term becomes quickly important, being the relevant contribution for densities above $\nu_{c}$. Both contributions are equal for $\nu \approx 0.30$.

\section{RESULTS}

In this section, results from several series of event driven simulations are presented. We consider a homogeneous, freely evolving system of elastic granular disks with periodic boundary conditions, implemented by means of the minimum image convention [35]. Systems with different numbers of particles and density have been simulated, and the dependence of the transport properties on these parameters has been investigated. A typical simulation started with a square lattice of particles having a Gaussian velocity distribution. After a transient period, the system reaches an equilibrium homogeneous state. Then, the measurement of the different properties of interest was carried out, using the procedure described in the previous section. For every calculation, the above process was repeated a number of times, typically 300 , in order to generate the ensemble average over different trajectories. Averages over different initial times were also considered for overriding the lack of statistical precision in the cases of the shear viscosity and the thermal conductivity, as compared with the self-diffusion coefficient. That implied performing longer simulations and store and handle the numerical data. The number of initial times used was in all cases larger than 200 . 


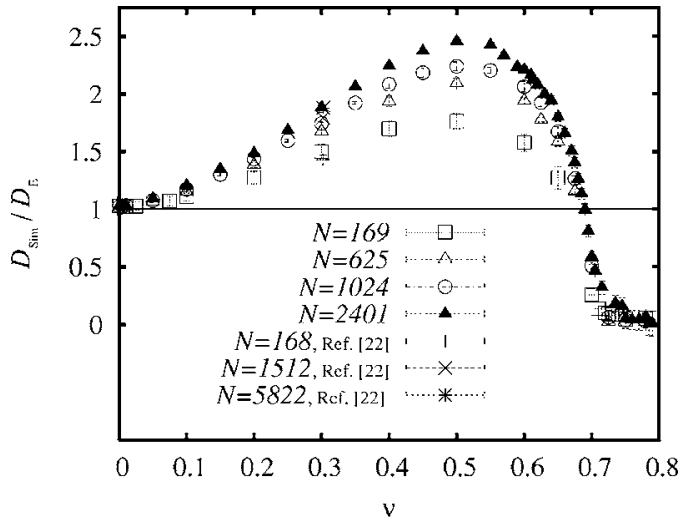

FIG. 2. Scaled self-diffusion coefficients for hard-disk fluids, as a function of the packing fraction $\nu$. For each value of the density, systems of $N=2401, N=1024, N=625$, and $N=169$ identical particles have been considered. Also included are previous results obtained by Holian et al. (in [22]), using nonequilibrium simulation techniques. Note that $D_{s i m} \cong D_{E}$ for $\nu=0.69$. The extrapolated tangent of the curve at this point crosses the line $D_{\text {sim }}=0$ at $\nu \approx 0.705$, a value which is fairly close to $\nu_{c} \approx 0.701$. [29]

\section{A. Self-diffusion coefficient}

As seen in Fig. 1 over the time scales simulated the mean square displacement is to a very good approximation linear in time. We find no evidence in the mean square displacement for the known logarithmic correction to the mean square displacement expected on the basis of hydrodynamic theory. Equation (3) can consequently be applied for the calculation of the self-diffusion coefficient.

In Fig. 2, the results obtained for the self-diffusion coefficient, $D_{\text {sim }}$, as a function of the solid fraction $\nu$ are presented. For each density, systems with different numbers of particles $N$ have been considered, namely $N=169,625,1024$, and 2401. Moreover, the reported values are the average over 300 independent trajectories. A strong deviation of $D_{\text {sim }}$ from the Enskog value $D_{E}$ is observed, even for relatively low densities. Also shown are some previous results obtained by Holian et al. (in [22]) at $\nu=0.3$ using a nonequilibrium molecular dynamics method. They consistently agree with the results reported here. For large enough packing fractions $\nu$, the particles become trapped in a crystalline lattice and no free movement is possible [36]. The self-diffusion coefficient must therefore vanish in this limit. This explains the rather fast decay to zero observed in the simulations. Of course, these high density effects are not captured by Enskog's theory.

The series of values of $D$ obtained for each value of $N$ in the interval $0<\nu \leqslant 0.5$ have been fitted to a third degree polynomial

$$
\frac{D_{\text {sim }}(\nu)}{D_{E}(\nu)}=a+b \nu+c \nu^{2}+d \nu^{3} .
$$

The values of the fitting coefficients are given in Table I, where it can be seen that the coefficient $a$, characterizing the dilute limit, seems to be weakly dependent on the number of particles used in the simulation. It is clearly larger than unity, indicating that the dilute limit is slightly underestimated by
TABLE I. Empirical fit of the simulation results for the selfdiffusion coefficient to the third order polynomial in Eq. (32). The error of the fitting for each value is of the order of the last figure given.

\begin{tabular}{lcccc}
\hline \hline $\mathrm{N}$ & $\mathrm{a}$ & $\mathrm{b}$ & $\mathrm{c}$ & $\mathrm{d}$ \\
\hline 169 & 1.0207 & 0.0433 & 8.629 & -11.43 \\
625 & 1.0299 & 0.3367 & 9.775 & -12.39 \\
1024 & 1.0259 & 0.4683 & 10.711 & -13.48 \\
2401 & 1.0287 & 0.5971 & 11.930 & -14.75 \\
\hline \hline
\end{tabular}

the expression for $D_{E}$ we have used. Moreover, we have carried out simulations with different boundary conditions and found always the same value consistently. In fact, similar deviations $(a>1)$ have been previously observed [37]. This can be due to the fact that the Enskog expression given by Eq. (5) has been computed in the first Sonine approximation. It is possible that the consideration of higher order polynomial corrections to the Sonine expansion would improve the agreement between theory and simulations in the low density limit.

Figure 2 and Table I clearly indicate a strong dependence of the fitted values of the self-diffusion coefficient on the system size. It should be expected that the simulation results converge to a well defined value as the number of particles increases, although this is not at all clear from Fig. 2, especially for densities around $\nu=0.5$. To check this convergence and characterize it, two series of simulations corresponding to $\nu=0.001$ and $\nu=0.5$ have been performed, see Fig. 4 . In the low density case, accurate size-independent results are already obtained with a small number of particles, namely with $N=169$. On the other hand, for $\nu=0.5$ the convergence is much slower, and reliable results require a few thousands of particles. More precisely, the dependence on $N$ in this case is quite well fitted by an exponential function $D_{\infty}-D_{0} \exp \left(-N / N_{0}\right)$, with $N_{0}=900$. The existence of an asymptotic value of $D_{\infty}$ follows from the above results at least in the time window accessible by the MD simulations.

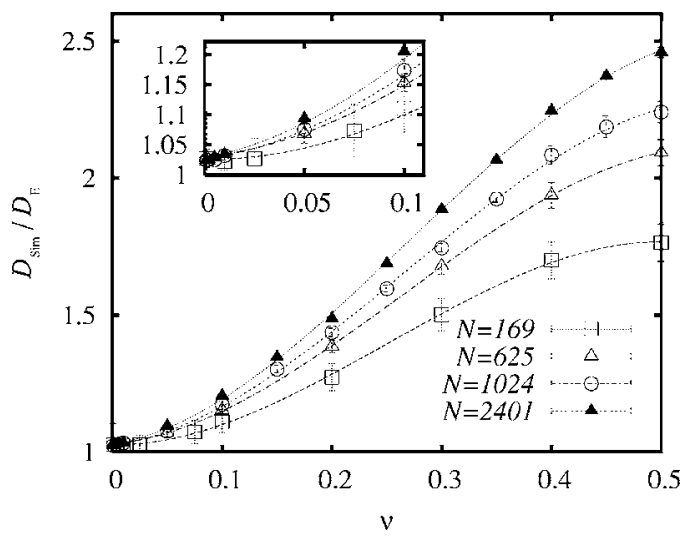

FIG. 3. Dependence of $D_{\text {sim }}$ on the solid fraction $\nu$ for low and moderate densities. The symbols are some of the simulation results given in Fig. 2. The lines are the fits to the polynomial (32) with the coefficients given in Table I. In the inset, the low density region is enlarged. 


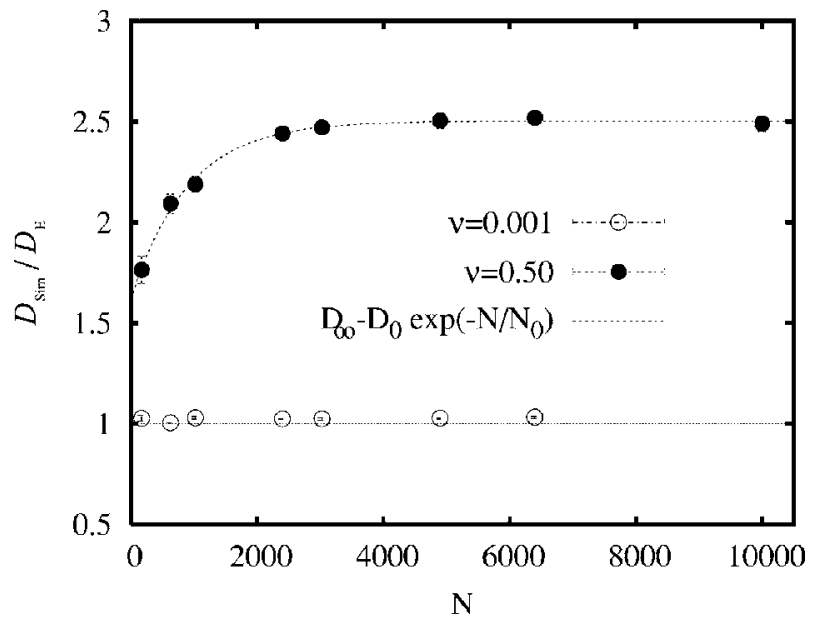

FIG. 4. Dependence of the measured self-diffusion coefficient on the number of particles $N$ for systems with densities $\nu=0.001$ and $\nu=0.5$, respectively. The results have been averaged over 30 trajectories for the system with $N=10000$ particles, and over 300 trajectories for all the other systems. The error bars in this scale are smaller than the symbols used.

The possibility of a much slower convergence to a higher value or even a divergence cannot be concluded from our data.

\section{B. Shear viscosity}

The simulation results for the shear viscosity at low and intermediate densities are shown in Fig. 5. The deviation from Enskog's theoretical prediction is typically below $10 \%$ up to the transition to the ordered state. This is true even for the simulations with the lowest number of particles. In fact, no strong dependence of the measured value of the transport coefficient on $N$ can be inferred from the simulation data in

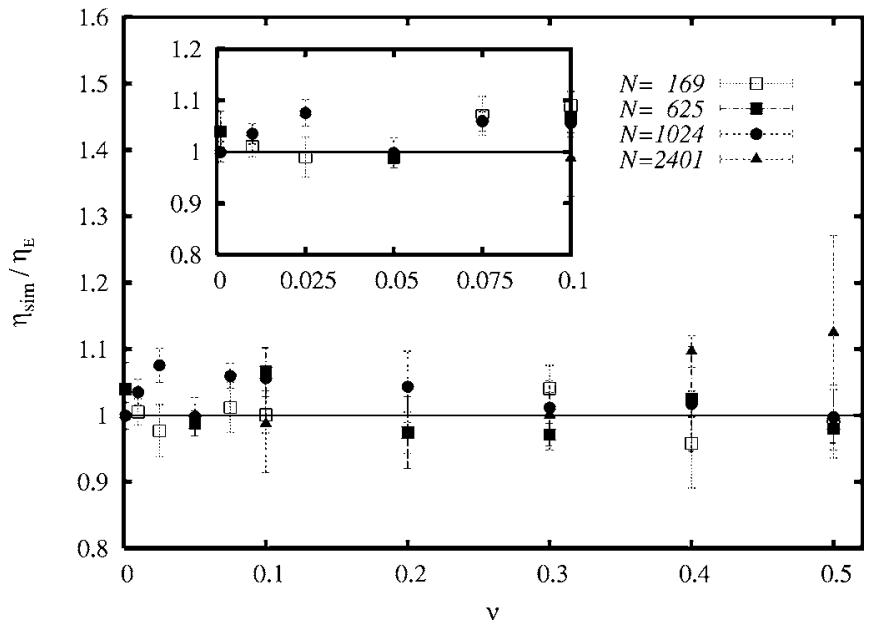

FIG. 5. Shear viscosity coefficient normalized with the Enskog prediction as a function of solid fraction for dilute and moderately dense systems for different $N$. Averages over 300 different initial conditions and over 300 different initial times have been taken. The low density region is amplified in the insert.

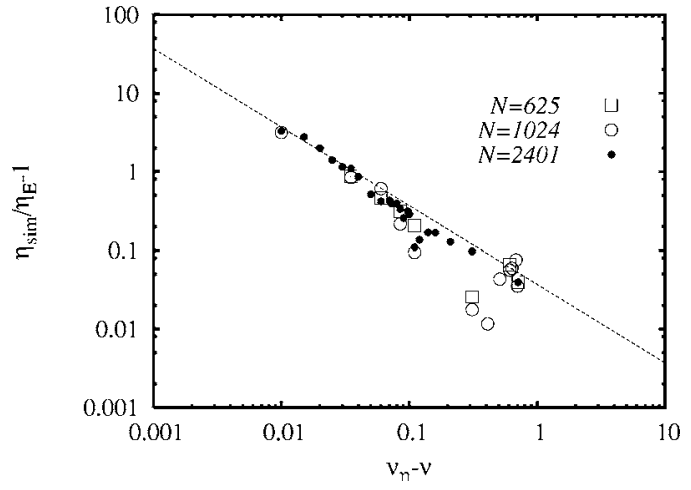

FIG. 6. Divergent behavior of the shear viscosity coefficient. The symbols show simulations with different numbers of particles, as indicated. They have been obtained by averaging as in Fig. 5 . The dashed line is the power-law fit given in Eq. (33).

this range. A similar behavior was found by Alder et al. for a system of hard spheres [20].

Enskog theory clearly underpredicts shear viscosity in the range $0.5<\nu<0.68$, contrary to what was found in the case of the self-diffusion coefficient, which dropped at $\nu_{c}$. This is so because the collision frequency in that range of densities is overestimated by Enskog's theory. In the crystalline region, the measurement of the shear viscosity becomes rather difficult, since the expected linear behavior of the increment in time of the dynamical variable $G_{\eta}(t)$ disappears-data not shown here [see Eq. (8)].

In Fig. 6 the deviations of the measured values of $\eta_{\text {sim }}$ from Enskog theory in the high density region are plotted in a logarithmic scale. A power-law divergent behavior of the viscosity is observed as the density approaches the critical value. Moreover, no shift of the critical viscosity $\nu_{\eta}$ is observed as the number of particles increases. The dashed line in the figure is the function

$$
\eta^{*}(\nu)=c\left(\nu_{\eta}-\nu\right)^{-1},
$$

with $c=0.037 \pm 0.001$, and $\nu_{\eta}=0.71 \pm 0.01$. This latter value approximately agrees with the density for which the selfdiffusion coefficient vanishes (see Fig. 2), and also with the critical (crystallization) density in the global equation of state proposed in [29]. Let us mention that for $N=169$ no linear behavior of $G_{\eta}$ was found for densities $\nu \gtrsim 0.65$ and, therefore, no results with this number of particles are included in Fig. 6 . The behavior of $\eta_{\text {sim }}$ in this region, in fact, seems to depend on $N$.

It is worth stressing at this point the relevance of making the measurements of the corresponding dynamical variable in the simulations with a frequency higher than the collision frequency in the fluid. In this case, the validity of the numerical procedures discussed in the previous section is guaranteed and the results obtained from them can be expected to be correct. On the other hand, if the time interval between successive measurements is increased and becomes comparable to the mean collision time of the system, the results for the time evolution of the corresponding dynamical variable $G$, can present a nonlinear behavior. And even if it turns out to be linear, the slope can lead to wrong values of 


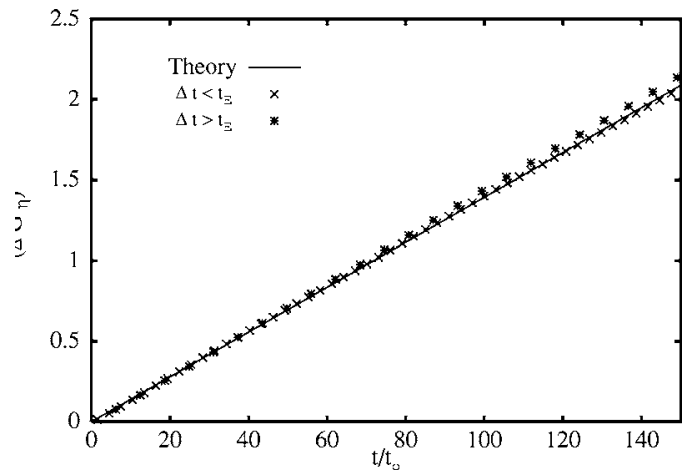

FIG. 7. Time evolution of the dynamical variable $\left(\Delta G_{\eta}\right)^{2}$ obtained with two different time intervals $\Delta t$, one shorter than the mean collision time $t_{E}$ and the other larger than it. The number of particles is $N=1024$ and the density $\nu=0.30$, in both cases. The solid line is the Enskog prediction. Time is scaled by $t_{0}$, Boltzman's mean time between collisions. Note that $t_{0}=t_{E}$ in the dilute limit, where $g_{2}(\sigma) \rightarrow 1$.

the corresponding transport coefficient. As an example, a comparison of the measurement of $G_{\eta}$ with two $\Delta t$ values is made on the same system of $N=1024$ particles and density $\nu=0.30$, see Fig. 7. More accurate results are obtained with a time step between measurements of $G_{\eta}$ shorter than the mean time between collisions. This is a consequence of the effect of multiple collisions that, if occurring between successive measurements of $G_{\eta}$, invalidate the arguments leading to Eqs. (11) and (14). Although this applies, in principle, to both the shear viscosity and the thermal conductivity, the simulation results show that the influence of the time step between the measurements employed is stronger for the former than for the latter.

\section{Thermal conductivity}

Figure 9 depicts the results obtained for the heat conductivity. Although the discrepancies with the Enskog predictions are not large (they remain of the order of 10\%), the qualitative behavior as a function of the density resembles that of the self-diffusion coefficient reported in Fig. 2. It exhibits a maximum around $\nu \simeq 0.55$, decaying below the Enskog prediction for larger densities $(\nu \geq 0.7)$. This decay is due to the increased mean free path due to the particle ordering. Of course, in contrast with $D_{\text {sim }}, \lambda_{\text {sim }}$ does not vanish in the ordered region, since there is still considerable transport of energy through collisions. The relative weight of the collisional part versus the kinetic component of $\lambda$ was shown in Fig. 8, which increases exponentially as density increases. Moreover, $G_{\lambda}$ was found to exhibit linear behavior in the transition to the ordered state, even for the smallest system considered $(N=169)$. Similarly to the case of the shear viscosity, no systematic dependence of the results on the number of particles used is observed. Let us mention that, although small, the deviations from the Enskog predictions in Fig. 9 are larger than those found by Alder et al. for a system of hard spheres [20].

In Fig. 10 we investigate the transition region $(\nu>0.65)$, where the dispersion of our measurements is clearly higher.

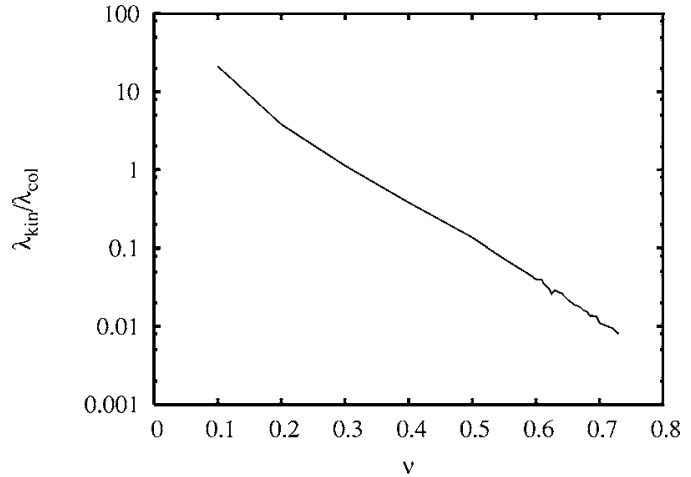

FIG. 8. Variation with density of the ratio between the kinetic and collisional contributions to the overall thermal conductivity, as calculated by Eqs. (29) and (31), respectively. The results correspond to a simulation with $N=2401$ particles. The behavior seems to be exponential, with a linear best fit curve $\lambda_{\text {kin }} / \lambda_{\text {col }} \approx 45 \cdot e^{-\nu / 0.083}$.

The deviation of the measurements with respect to Enskog's value is plotted using two different expressions for the pair correlation function $g_{2}(\sigma)$ set in Eq. (24). On the one hand, we have used the formula given in Eq. (6), and these are the open symbols. For the other two series (solid symbols) a semiempirical formula, valid in the whole range of densities studied here, was used as given in Ref. [29]:

$$
g_{2}^{L}(\sigma)=g_{2}(\sigma)+m\left(\nu \mid \nu_{c}, m_{0}\right)\left[\frac{P_{\text {dense }}(\nu)}{2 \nu}-g_{2}(\sigma)\right]
$$

where $m\left(\nu \mid \nu_{c}, m_{0}\right)$ is a connecting function, and $P_{\text {dense }}$ is the reduced pressure in the dense region (see Ref. [29] for more details). The explicit expressions of these functions are:

$$
m\left(\nu \mid \nu_{c}, m_{0}\right)=\frac{1}{1+\exp \left[-\left(\nu-\nu_{c}\right) / m_{0}\right]},
$$

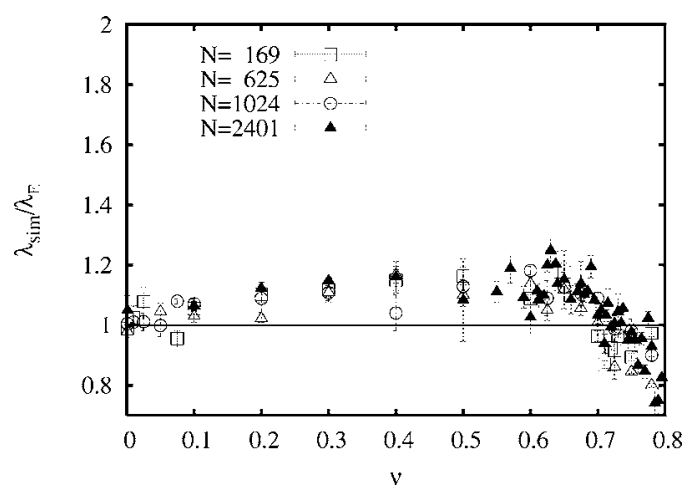

FIG. 9. Thermal conductivity coefficient as a function of the solid fraction $\nu$ for an elastic hard-disk systems of different numbers of particles $N$, as indicated. The values have been averaged in the same way as in Fig. 5. Error-bars are shown when larger than the symbols used. 


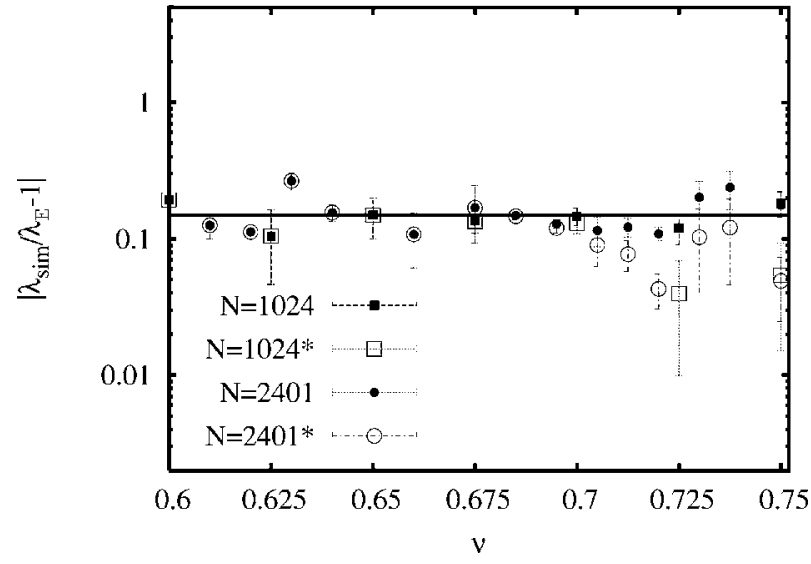

FIG. 10. Deviation of the values measured for the thermal conductivity coefficient, $\lambda_{\text {sim }}$, from Enskog's value, $\lambda_{E}$, as a function of the solid fraction $\nu$ for dense systems. The values have been averaged in the same way as in Fig. 5. For the scaling of the tagged series $N=1024^{*}$ and $N=2401^{*}$, the values $\nu_{c}=0.71$ and $m_{0}=0.01$ have been used. More details about the scaling of these results are given in the text. The solid line indicates the fixed value $\left(\lambda_{\text {sim }} / \lambda_{E}-1\right)=0.15$.

$$
P_{\text {dense }}(\nu) \approx \frac{2 \nu_{\eta}}{\nu_{\eta}-\nu}\left[1-0.04\left(\nu_{\eta}-\nu\right)+3.25\left(\nu_{\eta}-\nu\right)^{3}\right]
$$

in terms of the parameters $\nu_{c}, \nu_{\eta}$ and $m_{0} \approx 0.0111$.

In Fig. 10 results for high values of $\nu$ are plotted. The logarithmic scale is used in order to be consistent with Fig. 6. The empirical pair correlation function $g_{2}^{L}$ is expected to work better for values of the density around and above $\nu \approx \nu_{c}=0.70[29,38,39]$. The deviation from the theoretical value remains approximately constant for a wide range of solid fractions, covering the low and moderate densities. When $g_{2}^{L}$ is used, the deviation remains constant even beyond the transition to the dense configuration, while there is a clear deviation of the data if the simplified form of Eq. (6) is used. This is most clearly observed in the range $(0.65 \lesssim \nu \lesssim 0.74)$. The effect of the correction for smaller values of $\nu$ is nevertheless negligible.

\section{DISCUSSION}

In this paper, the transport coefficients of a system of elastic hard disks have been evaluated by means of equilibrium molecular dynamics simulations. In order to avoid the fundamental difficulties recently identified in the use of the standard Green-Kubo formulas in hard-particle systems [30], a Helfand-Einstein representation has been employed. The consideration of periodic boundary conditions in the simulation of a nonsheared, isotropic, homogeneous, freely evolving system of elastic hard spheres or disks forces some modifications of the usual Einstein-Helfand's formulas for the transport coefficients, especially if the minimum image convention is used. Moreover, the expressions proposed here are especially suitable for event driven methods. They allow a detailed study of the dependence of the coefficients on the system size and density, and also the relative weight of the collisional and kinetic contributions.

\section{A. Remarks on the long time tails}

For the sake of putting the results reported here into a proper context, some comments on the role played by the long time tails exhibited by the Green-Kubo time correlation functions [28] are required. For the sake of concreteness and also because it is by far the most studied transport coefficient, we will particularize the following discussion to the self-diffusion coefficient $D$. Its Green-Kubo representation involves the time integral of the velocity autocorrelation function (VACF). In 1970, Alder and Wainwright [27] reported from molecular dynamics simulations an algebraic long time tail of the VACF with an exponent $d / 2$, where $d$ is the dimensionality of the system. For $d=2$, the case considered here, this implies the presence of a contribution to $D$ of the form $a t_{0} \ln \frac{t}{t_{0}}$ in the time scale in which the algebraic decay holds. Here, $a$ is a constant measuring the amplitude of the tail contribution and $t_{0}$ the characteristic time at which it shows up.

Theoretical analysis of the long time tails has been carried out since its discovery by using kinetic theory and also mode coupling theory. A partial review is given in Ref. [40]. In particular, mode coupling theories have led to the prediction that the $t^{-1}$ decay for $d=2$ corresponds to intermediate times, while an asymptotic decay as $(t \sqrt{\ln t})^{-1}$ is expected at larger times [41]. Simulations in two and three-dimensional hard fluids $[42,43]$ are consistent with the predictions from a finite- $N$ mode-coupling theory for the VACF, although the statistical accuracy of the data does not allow a precise verification of the exponent of the algebraic tail or its amplitude.

More conclusive simulation results have been obtained in lattice-gas cellular automata. In this case [44], the $t^{-1}$ tail has been perfectly observed and it has been verified that its amplitude is predicted to within a few per cent by modecoupling theory. On the other hand, the asymptotic logarithmic tail mentioned above is expected to dominate only on time scales large compared with $10^{10}$ mean free times, what is fully irrelevant from a practical point of view [45].

Quite surprisingly, all discussions we are aware of in the literature identifying the time tails in computer simulations deal with the VACF, but we have not found any analysis of its influence on the observed values of the self-diffusion coefficient. The results reported in this paper, and also in Ref. [26], seem to indicate that the above influence remains negligible over a time scale going well into the time scale of the $t^{-1}$ tails (see Fig. 1). On this scale, $D$ and also the other transport coefficients analyzed, and given by the GreenKubo or Helfand expressions, appear as constant, the contributions from the tails remaining very small. To be more explicit, let us indicate that in the simulations with $N=2401$ particles reported in Fig. 2, the root mean square displacement was verified to exhibit a linear profile up to times of the order of 8000 collision times. The conclusion is that usual Navier-Stokes hydrodynamics, with time-independent transport coefficients, works on such time scales. What happens at the far end of the observed plateau for the transport coeffi- 
cient remains open, in our opinion, except for theoretical predictions. Let us point out that the above picture is consistent with the simulation results for the VACF reported in [44]. An analysis of them indicates that the influence of the $t^{-1}$ tail on the self-diffusion transport coefficient is still only of the order of one percent at $t \sim 1000$ collision times.

\section{B. Discussion on our results for the transport coefficients}

We start by the self-diffusion coefficient $D$. The Enskog approximation leads in this case to values that underestimate the simulation results by factors up to two, for moderate values of the density $(\nu \leqslant 0.3)$, the discrepancies being already relevant at rather low densities. The observed density dependence of the transport coefficient is well fitted by a third order polynomial for $\nu \leqslant 0.5$, with coefficients that slightly depend on the number of particles, $N$, of the system. It has been verified that, at least in the hydrodynamic limit accessible by the MD simulations, $D$ tends to a well defined limit as $N$ becomes large enough. At higher densities, the transition liquid-solid is clearly depicted in the behavior of the self-diffusion coefficient. It rapidly falls to zero as a consequence of the caging of the particles. Finite size effects are more relevant for dense systems, in which the self-diffusion coefficient approaches its asymptotic value exponentially with $N$.

For the shear viscosity the dependence of the results on the size of the system is much smaller. Also much weaker deviations from the Enskog prediction are observed at low and intermediate densities. Nevertheless, closer to the gassolid transition, a power law divergent behavior has been identified. Interestingly, the density value for which the viscosity would become eventually singular $\left(\nu_{c} \simeq 0.71\right)$, agrees with the density at which the system begins to show an ordered triangular structure [46-48]. There were no data obtained for the shear viscosity above $\nu_{\eta}$.

Note that the results presented here refer to "non-sheared" systems. We have avoided therefore the problem of the system becoming inhomogeneous and developing a shearband [49]. A sheared system will not show the divergence found for the viscosity because of the shearbanding instability [52].

The pressure also diverges but at a considerably higher density $\nu_{\max } \approx 0.9069$. At the crystallization density $\nu_{c} \approx \nu_{\eta}$ $\approx 0.7$, both pressure and heat conductivity show a drop relative to the Enskog prediction due to the better ordering in the crystalline phase (see Fig. 11). The use of a more elaborate expression of the pair correlation function, valid in a wider range of densities than Henderson's approximation, improves the agreement of our data with Enskog theory.

\section{Conclusion}

In summary, we have found Enskog theory working rather well for pressure, heat conductivity, and shear viscosity well

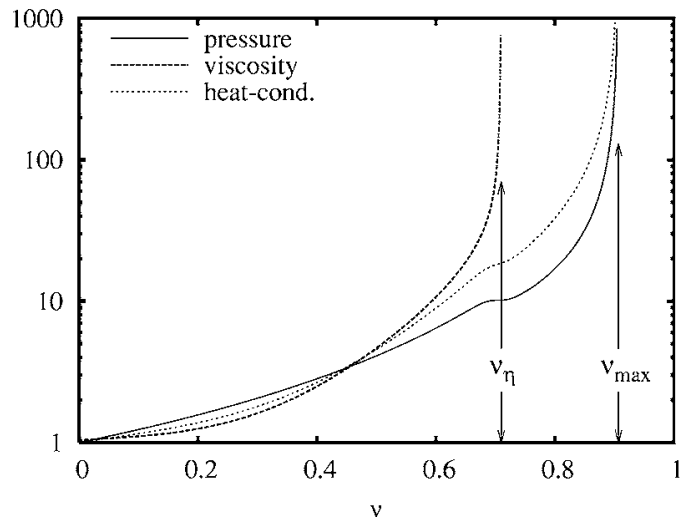

FIG. 11. Schematic plot of the transport coefficients. The solid line gives the pressure $P=1+2 \nu g_{L}$, with $g_{L}$ from Eq. (35); the dashed line gives the scaled shear viscosity, i.e., Eq. (16), where $g_{L}$ is used instead of $g_{2}$, and multiplied by our empirical correction factor $1+c_{\eta}$ in Eq. (34); and the dimensionless heat conductivity from Eq. (31), also with $g_{L}$ used instead of $g_{2}$.

below the crystallization density $\nu_{c}$. When the Enskog expressions are corrected by an appropriate pair correlation function $g_{L}$, which accounts for the better ordering in the crystalline phase, the theory performs well for pressure and heat conductivity up to the maximal possible density $\nu_{\max }$.

Only the shear viscosity shows a power-law divergence at $\nu_{\eta} \approx \nu_{c}$ with values above Enskog theory already becoming visible at intermediate densities, $\nu>0.5$. Thus, shear viscosity behaves differently than the other transport coefficients studied. Its divergence, implying that the shear modes are hindered for $\nu>\nu_{\eta}$. This could in fact be understood as one reason for shear-band formation. A sheared system at high densities typically splits into shear bands (with lower density) and a compressed dense crystal (with correspondingly higher density). From a different point of view, our observations are also consistent with the concept of dilatancy: A dense packing with $\nu>\nu_{\eta}$ can only be sheared by first experiencing dilatancy so that $\nu$ drops below $\nu_{c}$.

\section{ACKNOWLEDGMENTS}

The research of J.J.B. was supported by the Ministerio de Educación y Ciencia (Spain) through Grant No. FIS200501398 (partially financed by FEDER funds). He also acknowledges J.W. Dufty for his many clarifying comments. S.L. acknowledges helpful discussion with J.T. Jenkins and M. Alam, as well as financial support of the DFG (Deutsche Forschungsgemeinschaft, Germany) and FOM (Stichting Fundamenteel Onderzoek der Materie, The Netherlands) as financially supported by NWO (Nederlandse Organisatie voor Wetenschappelijk Onderzoek). 
[1] L. D. Landau and E. M. Lifshitz, Fluid Mechanics (AddisonWesley, Reading, MA, 1981).

[2] P. Résibois and M. de Leener, Classical Kinetic Theory of Fluids (Wiley-Interscience, New York, 1977).

[3] J. A. McLennan, Introduction to Nonequilibrium Statistical Mechanics (Prentice Hall, Englewood Cliffs, NJ, 1989).

[4] R. Zwanzig, Annu. Rev. Phys. Chem. 16, 67 (1965).

[5] E. Helfand, Phys. Rev. 119, 1 (1960).

[6] M. P. Allen and D. J. Tildesley, Computer Simulation of Liquids (Clarendon Press, Oxford, 1993).

[7] Physics of dry granular media, edited by H. J. Herrmann, J.-P. Hovi, and S. Luding (Kluwer Academic Publishers, Dordrecht, 1998).

[8] Granular Gases, edited by T. Pöschel and S. Luding (SpringerVerlag, Berlin, 2000).

[9] N. V. Brilliantov and T. Pöschel, Kinetic Theory of Granular Gases (Oxford University Press, Oxford, 2004).

[10] J. J. Brey, J. W. Dufty, C. S. Kim, and A. Santos, Phys. Rev. E 58, 4638 (1998); J. J. Brey and D. Cubero, in Granular Gases, edited by T. Pöschel and S. Luding (Springer-Verlag, Berlin, 2001).

[11] J. W. Dufty and J. J. Brey, J. Stat. Phys. 109, 433 (2002).

[12] J. J. Brey, J. W. Dufty, and M. J. Ruiz-Montero, in Granular Gas Dynamics, edited by T. Pöschel and N. Brilliantov (Springer-Verlag, New York, 2003).

[13] V. Garzó and J. W. Dufty, Phys. Rev. E 59, 5895 (1999).

[14] J. F. Lutsko, Phys. Rev. E 72, 021306 (2005).

[15] J. W. Dufty and V. Garzó, J. Stat. Phys. 105, 723 (2001).

[16] J. W. Dufty, J. J. Brey, and J. Lutsko, Phys. Rev. E 65, 051303 (2002).

[17] I. Goldhirsch and T. P. C. van Noije, Phys. Rev. E 61, 3241 (2000).

[18] J. W. Dufty, A. Baskaran, and J. J. Brey, e-print cond-mat/ 0507609.

[19] J. T. Jenkins and S. Savage, J. Fluid Mech. 130, 187 (1983); J. T. Jenkins and M. W. Richman, Phys. Fluids 28, 3485 (1985).

[20] B. J. Alder, D. M. Gass, and T. E. Wainwright, J. Chem. Phys. 53, 3813 (1970).

[21] B. J. Alder and T. E. Wainright, Phys. Rev. A 1, 18 (1970).

[22] J. J. Erpenbeck and W. W. Wood, in Statistical Mechanics Part $B$, edited by B. J. Berne (Plenum, New York, 1977).

[23] J. P. Hansen and I. A. McDonald, Theory of Simple Liquids (Academic Press, London, 1986).

[24] J. J. Erpenbeck, and W. W. Wood, Phys. Rev. A 43, 4254
(1991).

[25] J. M. Montanero, and A. Santos, in Rarefied Gas Dynamics, edited by R. Brun, R. Campargue, R. Gatignol, and C. Legrand (Cepadues, Tolouse, 1999).

[26] S. Viscardy and P. Gaspard, Phys. Rev. E 68, 041204 (2003).

[27] B. J. Alder and T. E. Wainwright, Phys. Rev. A 1, 18 (1970).

[28] J. R. Dorfman, in Fundamental Problems in Statistical Mecahnics, edited by E. D. G. Cohen (North-Holland, Amsterdam, 1975), Vol. 3, p. 277.

[29] S. Luding, Phys. Rev. E 63, 042201 (2001).

[30] J. W. Dufty, Mol. Phys. 100, 2331 (2002); J. W. Dufty and M. H. Ernst, ibid. 102, 2123 (2004).

[31] D. M. Gass, J. Chem. Phys. 54, 1898 (1971).

[32] D. Henderson, Mol. Phys. 30, 971 (1975).

[33] J. J. Erpenbeck, Phys. Rev. E 51, 4296 (1995).

[34] C. S. Campbell, Annu. Rev. Fluid Mech. 22, 57-92 (1990).

[35] B. D. Lubachevsky, Int. J. in Computer Simulation 2, 373 (1992).

[36] B. J. Alder and T. E. Wainwright, Phys. Rev. 127, 359 (1962).

[37] J. J. Brey, M. J. Ruiz-Montero, D. Cubero, and R. GarcíaRojo, Phys. Fluids 12, 876 (2000).

[38] S. Luding, Adv. Complex Syst. 4(4), 379 (2002).

[39] S. Luding and A. Santos, J. Chem. Phys. 121, 8458 (2004).

[40] Y. Pomeau and P. Résibois, Phys. Rep., Phys. Lett. 19C, 64 (1975).

[41] D. Forster, D. R. Nelson, and M. J. Stephen, Phys. Rev. A 16, 732 (1977).

[42] J. J. Erpenbeck and W. W. Wood, Phys. Rev. A 26, 1648 (1982).

[43] J. J. Erpenbeck and W. W. Wood, Phys. Rev. A 32, 412 (1985).

[44] D. Frenkel and M. H. Ernst, Phys. Rev. Lett. 63, 2165 (1989).

[45] T. Naitoh, M. H. Ernst, and J. W. Dufty, Phys. Rev. A 42, 7187 (1990).

[46] J. Tobochnik and P. M. Chapin, J. Chem. Phys. 88, 5824 (1988).

[47] S. Luding and O. Strauss, in Granular Gases, edited by T. Pöschel and S. Luding (Springer, Berlin, 2000).

[48] S. Torquato, T. M. Truskett, and P. G. Debenedetti, Phys. Rev. Lett. 84, 2064 (2000).

[49] M. Alam and S. Luding, Phys. Fluids 17, 063303 (2005).

[50] E. Khain and B. Meerson, Phys. Rev. E (to be published).

[51] V. Kumaran, Phys. Rev. Lett. 96, 258002 (2006).

[52] S. Luding (unpublished). 